\title{
Importance of synovial fluid aspiration when injecting intra-articular corticosteroids
}

\author{
Tomas Weitoft, Per Uddenfeldt
}

\begin{abstract}
Objective-The aim of this prospective study was to find if a complete synovial fluid aspiration before injecting intraarticular corticosteroids influences the treatment result.

Methods-The study was performed in 147 patients with rheumatoid arthritis (RA). One hundred and ninety one knees with synovitis were randomised to arthrocentesis $(n=95)$ or no arthrocentesis $(\mathrm{n}=96)$ before $20 \mathrm{mg}$ triamcinolone hexacetonide was injected. The duration of effect was followed up for a period of six months. All patients were instructed to contact the rheumatology department if signs and symptoms from the treated knee recurred. If arthritis could be confirmed by a clinical examination a relapse was noted.

Results-There was a significant reduction of relapse in the arthrocentesis group $(\mathrm{p}=0.001)$.

Conclusion-The study shows that aspiration of synovial fluid can reduce the risk for arthritis relapse when treating RA patients with intra-articular corticosteroids. It is concluded that arthrocentesis shall be included in the intra-articular corticosteroid injection procedure.

(Ann Rheum Dis 2000;59:233-235)
\end{abstract}

The society and the medical profession are more and more requiring a rationale and evidence based medicine. Ineffective methods should be reduced to diminish health care costs. It is therefore essential that common routines in the clinical practice are studied.

Intra-articular corticosteroid treatment is a frequently used method to relieve arthritic symptoms. The injection procedure varies between physicians, for instance concerning synovial fluid aspiration before the corticosteroid is injected. In the literature there are diverging opinions on this matter. ${ }^{1-3}$

Accepted for publication 1 December 1999

Table 1 Patient characteristic

\begin{tabular}{lll}
\hline & $\begin{array}{l}\text { Arthrocentesis group } \\
(n=95)\end{array}$ & $\begin{array}{l}\text { No arthrocentesis group } \\
(n=96)\end{array}$ \\
\hline Sex (male/female) & $22 / 73$ & $25 / 71$ \\
Mean age (y) & $58(22-81)$ & $60(19-82)$ \\
Mean disease duration (y) & $13(1-54)$ & $13(1-55)$ \\
Continuous NSAID treatment & 77 & 70 \\
DMARD treatment & 74 & 80 \\
Prednisone <7.5 mg/day & 19 & 19 \\
Mean ESR $(\mathrm{mm} \mathrm{1st} \mathrm{h)}$ & $52(6-119) \mathrm{n}=73$ & $42(4-112) \mathrm{n}=78$ \\
Mean s-CRP $(\mu \mathrm{mol} / \mathrm{l})$ & $44(5-166) \mathrm{n}=72$ & $38(5-165) \mathrm{n}=83$
\end{tabular}

NSAID $=$ Non-steroidal anti-inflammatory drug. DMARD $=$ Disease modifying anti-rheumatic drug. $\mathrm{ESR}=$ erythrocyte sedimentation rate. $\mathrm{s}-\mathrm{CRP}=$ serum $\mathrm{C}$ reactive protein.
The scientific documentation concerning the optimal injection procedure is sparse. A complete aspiration of synovial fluid is time consuming and if it does not influence the treatment result, it might be excluded. The aim of this study was to find if the arthrocentesis before the injection influences the duration of the clinical effect.

\section{Methods}

Consecutive patients fulfilling the 1987 ACR criteria for rheumatoid arthritis (RA) ${ }^{4}$ and with signs and symtoms of knee joint arthritis (heat, tenderness, swelling and effusion) were at our outpatient rheumatology department asked to participate in this study. Patients in function class 4 according to Steinbrocker, ${ }^{5}$ patients with daily oral corticosteroid treatment corresponding to $7.5 \mathrm{mg}$ prednisone or more, and patients with intra-articular corticosteroid treatment in the knee in the past three months, were excluded.

The included knees were randomised according to the patient's date of birth to arthrocentesis or no arthrocentesis before $20 \mathrm{mg}$ triamcinolone hexacetonide was injected into the inflamed knee joint. The joints in the arthrocentesis group were aspirated of as much synovial fluid as possible. In the no arthrocentesis group they were aspirated just to confirm existance of effusion, but the fluid was not removed from the joint. The needle size was $0.7 \mathrm{~mm} \times 40 \mathrm{~mm}$. All patients were recommended to have 24 hour rest at home after the injection.

A total of 191 knees on 147 patients were included in the study, 144 female and 47 male. Ninety five knees were randomised to arthrocentesis and 96 had no arthrocentesis. Table 1 gives the patient characteristics.

The patients were told to contact the rheumatology department if no relief or when symptoms from the treated knee recurred. When that happened the knee was examined, and if there was signs of arthritis a relapse was noted, and the patient was offered another corticosteroid injection. We assessed that the other patients, with no need for further examination and injection, had no relapse of clinical relevance.

All patients were followed up for six months, mostly with follow up visits, otherwise a phone call to confirm that patients with knee joint symptoms had not been visiting other physicians and that no unknown relapses had occurred.

Log rank test was used to test for significant difference between the groups during the observation period, and $\chi^{2}$ test when proportions of relapse in the two groups were 


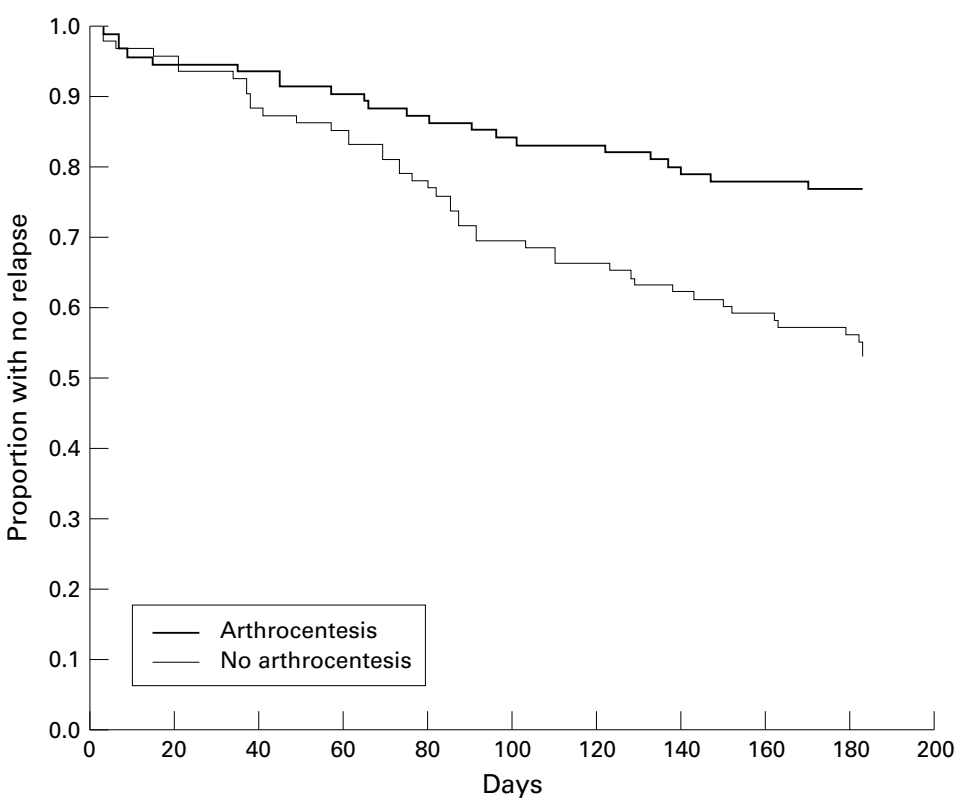

Figure 1 Duration of corticosteroid injection effect with or without arthrocentesis.

compared at the end point of the study. A p value of $<0.05$ was regarded as significant.

\section{Results}

One patient in the no arthrocentesis group moved from the district and was lost for follow up.

The proportion of relapses in the arthrocentesis group during the observation period was significantly reduced $(p=0.0009)$ and at the end of the study there were $23 \%$ relapses in the arthrocentesis group and $47 \%$ in the no arthrocentesis group $(p=0.001)$. A subgroup analysis of unilateral knee joint injections showed the same results $(p=0.02)$. Figure 1 gives the complete results.

The mean aspirated synovial fluid volume in the arthrocentesis group was $14 \mathrm{ml}$ (range 0-147 ml). The intention was to aspirate synovial fluid from all knees in the group, but in eight cases it was not possible to get any fluid before the injection. Six of them had no relapse during the observation time.

During the observation period the disease modifying anti-rheumatic drug treatment was changed in 22 cases in the arthrocentesis group and in 25 cases in the no arthrocentesis group.

\section{Discussion}

Many studies on the response of intra-articular corticosteroid treatment include patients with different types of arthritic disorders. ${ }^{67}$ We think that this is not appropiate, as the inflammatory process is different in erosive and nonerosive diseases. We have chosen just to study patients with RA, to have as homogenous population as possible.

From the patient's view a relapse occurrs when symptoms from the treated knee reappear. As the purpose for giving the corticosteroid injection is to relieve arthritic symptoms, we have in our study used the clinical response followed by an examination to confirm a relapse as arthritis. The method has been used in earlier investigations ${ }^{89}$ and we think it is a both clinically relevant and an easy way to measure the duration of response.

Several investigators have been looking for factors predicting good response..$^{10}{ }^{11}$ Neither disease duration, radiological scores, joint counts or blood levels of inflammatory parameters, such as erythrocyte sedimentation rate and serum $\mathrm{C}$ reactive protein correlate to efficacy of intra-articular corticosteroids. This was borne in mind when designing the study. Only a high percentage of synovial fluid polymorphonuclear leucocytes seems to relate to better results. ${ }^{11}$ This was not analysed, as synovial fluid was not available in both groups.

This is to our knowledge the first study comparing a complete aspiration and intraarticular injection with injection alone. The lack of studies may explain why the recommendations in the literature differ. Neustadt ${ }^{1}$ recommends aspiration of as much synovial fluid as possible to reduce the intra-articular pressure, to decrease the potentially deleterious effects of destructive enzymes in the synovial fluid and to diminish the dilution factor of the corticosteroid. Williams and Gumpel ${ }^{2}$ think that there should be synovial fluid left in the joint, so as to permit free diffusion of corticosteroid around the cavity. Dixon and Emery ${ }^{3}$ also recommend aspiration, but do not think it is essential. Their experiences are that arthrocentesis can give an immediate reduction of pain and that the corticosteroid effect may appear earlier.

The synovial fluid contains a variety of destructive enzymes, proinflammatory substances and cartilage degradation products, which are removed from the joint by aspiration. If this mechanism is important for the more long acting corticosteroid effect after arthrocentesis, joint lavage could further improve the result. Previous studies are short-term, but show a slight additive effect of joint lavage, ${ }^{12}{ }^{13}$ which supports this hypothesis.

A large effusion may be painful and another reason for aspirating synovial fluid is that the arthrocentesis can give a fast pain relief and increase in the range of motion.

A report by Jones et $a l^{14}$ shows how uncertain is the placement of the injection. The authors used a radiological evaluation of intra-articular injections with a corticosteroid mixed with a contrast medium given in various joints. A large proportion of the injections turned out to be extra-articular instead. In our study it was not possible to aspirate synovial fluid from eight knees in the arthrocentesis group. Those injections might have been extra-articular. This is in accordance with a recent study by Bliddal $^{15}$ showing that $9 \%$ of knee joint injections were extra-articular.

The result of our prospective randomised study comparing a complete synovial fluid aspiration and intra-articular corticosteroid injection with injection alone indicates, that the arthrocentesis reduces the risk for arthritis relapse in RA patients. We conclude that synovial fluid aspiration, though time consuming, should be included in the intra-articular corticosteroid injection procedure. 
Hans Högberg has been of great help in statistical analysis. We also wish to thank Dr Shirani Jayawardena and Dr Henrik Schiller for their help in including patients in the study.

Funding: this study was supported by FOU-forum, Landstinget Gävleborg.

1 Neustadt DH. Intraarticular therapy in rheumatoid synovitis of the knee: effects of postinjection rest regimen. Clin Rheumatol Practice 1985;3:65-8.

2 Williams P, Gumpel M. Aspiration and injection of joints (1). BMJ 1980;281:1990-2.

3 Dixon ASJ, Emery P. Local injection therapy in rheumatic diseases. 4th ed. Basel: Eular Publishers 1992.

4 Arnett FC, Edworthy SM, Bloch DA, Shane DA, Fries JF, Cooper NS, et al. The American Rheumatism Association 1987 revised criteria for the classification of rheumatoid arthritis. Arthritis Rheum 1988;31:315-24.

5 Steinbrocker O, Traeger CH, Betterman RC. Therapeutic criteria in rheumatoid arthritis. JAMA 1949;140:659-62.

6 McCarty D. Treatment of rheumatoid joint inflammation with triamcinolone hexacetonide. Arthritis Rheum 1972; 15:157-73.

7 Chakravarty K, Pharoah P, Scott DGI. Controlled study of postinjection rest following intra-articular steroid therapy for knee synovitis. Br J Rheumatol 1994;33:464-8.
8 Jalava S, Virsiheimo B. Triamcinolone hexacetanide in the treatment of therapy-refractory gonitis. J Int Med Res treatment of

9 Padeh S, Passwell H. Intraarticular corticosteroid injection in the management of children with chronic arthritis. Arthritis Rheum 1998;7:1210-14

10 Jones A, Doherty M. Intra-articular coticosteroids are effective in osteoarthritis but there are no clinical predictors of response. Ann Rheum Dis 1996;55:829-32.

11 Luukkiainen R, Hakala M, Sajanti E, Huhtala H, YliKerttula U, Hämenkorpi R. Predictive value of synovial fluid analysis in estimating the efficacy of intra-articular corticosteroid injections in patients with rheumatoid arthritis. Ann Rheum Dis 1992;51:874-6.

12 Fitzgerald O, Hanly J, Callan A, McDonald K, Molany J, Breshnihan B. Effects of joint lavage on knee synovitis in Breshnihan B. Effects of joint lavage on knee synov
rheumatoid arthritis. Br J Rheumatol 1985;24:6-10.

13 Srinavasan A, Amos M, Webley M. Effects of joint washout and steroid injection compared with either washout or steroid injection alone in rheumatoid knee effusion. Br J Rheumatol 1995;34:771-3

14 Jones A, Regan M, Ledingham J, Pattrick M, Manhire A, Doherty M. Importance of placement of intra-articular steroid injections. BMJ 1993;307:1329-30.

15 Bliddal $\mathrm{H}$. Placement of intraarticular injections verified by mini air-arthrography. Ann Rheum Dis 1999;58:641-6. 